When we turn to a more realistic theory such as quantum electrodynamics, we are faced with the problem of diagonalizing a much more complicated Hamiltonian. This is done in principle via the asymptotic in and out fields ${ }^{3}$ and will result in practice (in perturbation theory) by inserting the solutions of the field equations as power series expansions in terms of the in or out fields. (From the asymptotic condition, $H$ is the time translation operator for the in and out fields. The only form of $H$ expressible as a polynomial in either the in or out creation and annihilation operators must then be the usual diagonal expression for the free energy in terms of these operators.) In the presence of bound states, we do not expect these series to converge and the complote diagonalization will involve a self-consistent type of calculation of these bound states. Evon in their absence the convergence is unlikely.

The diagonalization process cannot involve a unitary transformation on the field operators. This is evident for the model discussed earlier and is also true for any relativistic theory as follows from Haag's theorem 4 . Indeed, the Heisenberg operators at finite $t$ and the corresponding Schrödinger operators must belong to a representation of the $\mathrm{CCR}^{5}$ which does not possess a no-particle state. These 'strange' representations are very complicated and lead to great difficulties in the $S$ - and $H$-pictures. In fact, all numerical successes of quantum ficld theory have been obtained by using Green's functions equations (GFE) These GFE avoid finding the strange representations by considering matrix elements of the field operators and not the operators themselves. We do not know yet whether there exist solutions to these $\mathrm{GFE}^{7}$; if we could show that they exist then we would have achieved the diagonalization process outside of perturbation theory.

In conclusion, we see no logical inconsistency in the foundation of quantum field theory provided the nonperturbative solutions of the GFE exist.

J. M. SrMON

J. G. TAYLOR

Department of Physics,

Rutgers, the State University,

New Brunswick, New Jersey.

1 Dirac, P. A. M., Nature, 203, 115 (1964).

'Van Hove, L., Physica, 18, 145 (1952). Coester, F., and Haag, R., Phys. Rev., 117, 1137 (1960). Araki, H., J. Math. Phys., 1, 492 (1960).

${ }^{3}$ Lehmann, H., Symanzik, K., and Zimmermann, W., Nuovo Cimento, 1, 205 (1955).

${ }^{4}$ Haag, R., Phys, Rev.,112, 669 (1958), Ruelle, D. Helv, Phys. Acta, 35, 147 (1962). Hepp, K., Ann. Phys. Assoc., 7, 85 (1963).

${ }^{5}$ Garding. L., and Wightman, A. S., Proc. V.S. Nat. Acad. Sei., 40, 617, 622 (1954). Segal, Amer. Math. Soc., 88, 12 (1958).

- Feynman, R. P., Phys. Rev., 76, 749 (1949). Dyson, F., Phys. Rev., 75, 1736 (1949), Schwinger, J., Phys. Rev., 73, 416 (1948).

Taylor, J. G., Nuovo Cimento supplement and On the Existence of Solutions of Field Equations, Proc. Third Eastern U.S. Theor. Phys. Conf. (October 1964).

\section{Precursor Shocks produced by a Large-yield Chemical Explosion}

Precursor shocks are phenomena normally associated with surface burst or low-level nuclear explosions. The precursor moves out along the ground ahead of the primary shock, and usually produces a large amount of airborne dust. The precursor has been explained ${ }^{1}$ as an effect of the intense thermal radiation produced by a nuclear explosion.

In July 1964, a 500 ton TNT hemispherical surface burst charge was detonated at Suffield Experimental Station in Alberta, Canada. High-speed photographs of the explosion show that in some radial directions dust clouds moved out ahead of the main shock and had reached a height of $50 \mathrm{ft}$. before its arrival. The dust clouds were enveloped by a shock wave. At ground-level this procursor eventually became downward facing and produced a reflected shock and a Mach stom. Photography from an aeroplane at $19,000 \mathrm{ft}$. immediately abovo the explosion showed that all the precursors were produced along wellcompacted roadways running radially from the charge centre. The precursors occurred in the region 250-750 ft. from the centre of the explosion, corresponding to peak overpressure levels of $150 \mathrm{lb}$./in. ${ }^{2-20} \mathrm{lb}$./in. ${ }^{2}$. A gauge measuring the total density within the blast wave, by means of a 3 -radiation absorption technique ${ }^{2}$, showed the dust density to be four times that of the peak air density expected in the blast wave at that position. Targets placed in the regions of the precursors experienced eonsiderably more damage than had been expected. From the evidence of seismometer records it seems probable that the precursors were produced by strong ground waves feeding energy into the air ahead of the air shock in a manner similar to that obsorved by Boys $^{3}$ for supersonic missiles penetrating metal plates and by Benioff, Ewing and Press ${ }^{4}$ for earthquakes.

Suffield Experimental Station,

JoHN M. DEWEY

Ralston, Alberta, Canada.

${ }^{1}$ Glasstone, S., edit., The Effects of Nuclear Weapons (U.S. Atomic Energy Commission, 1962).

2 Dewey, J. M., and Anson, W. A., J. Sci. Instrum., 40, 568 (1963).

3 Boys, C. V., Nature, 47, 440 (1893).

4 Benioff, H., Ewing, M., and Press, F., Proc. U.S. Nat. Acad. Sci., 5, 37, 600 (1951).

\section{Does Quantum Mechanics exclude Life?}

P. T. LANDSBERG, in a recent paper ${ }^{1}$, reopened a problem discussed earlier by Wigner: ${ }^{2}$ the possibility that quantum mechanics might predict and explain the phenomena of life.

More precisely, this problem may be formulated by asking two questions which sum up its essential content. These derive from a recognition that a living organism, together with the environment from which it draws its food, may be considered as a quantum system of very large size, which we may assume to be isolated from any external influence.

Let us start by supposing that such a system contains initially no living organism. What, then, is the probability that it will evolve towards a state in which a living organism is present? This is the problem of the spontaneous generation of life.

Alternatively, let us suppose that our system contains initially one living organism. What, then, is its probability of evolving toward a state in which two such organisms are present? This is the problem of reproduction.

Wigner sought, essentially, to reply to the second of these two questions: he thus assumed reproduction to be a characteristic and definitive property of life. The conclusion he reached, however, on the basis of a statistical treatment, was that quantum mechanics predicts a practically nil probability for the existence of states corresponding to auto-duplication of a part of the system. He therefore suggested that quantum mechanies is not suitable for a complete description of all natural phenomena or that, at the least, it must be modified to include concepts, such as consciousness, which are not necessary for the description of physical phenomena.

Landsberg deliberately avoids giving any precise definition of a living organism, limiting himself to the assumption that the number of dimensions of the region of phase space, corresponding to the presence of $n$ living organisms in the system, diminishes very rapidly as $n$ increases. Using loss-restrictive statistical assumptions than Wigner, he establishes that spontaneous generation of lifo and reproduction are not completely ruled out by quantum mechanics, although their probability on average (that is, over a large number of possible initial conditions) remains extremely small. However, favourable initial conditions for self-reproduction can certainly be expected. 